\title{
Long-Lasting Protection of Activity of Nucleoside Reverse Transcriptase Inhibitors and Protease Inhibitors (PIs) by Boosted PI Containing Regimens
}

\author{
Alexandra U. Scherrer ${ }^{1 *}$, Jürg Böni ${ }^{2}$, Sabine Yerly ${ }^{3}$, Thomas Klimkait ${ }^{4}$, Vincent Aubert ${ }^{5}$, \\ Hansjakob Furrer ${ }^{6}$, Alexandra Calmy ${ }^{7}$, Matthias Cavassini ${ }^{8}$, Luigia Elzi ${ }^{9}$, Pietro L. Vernazza ${ }^{10}$, \\ Enos Bernasconi ${ }^{11}$, Bruno Ledergerber ${ }^{1}$, Huldrych F. Günthard ${ }^{1}$, the Swiss HIV Cohort Study (SHCS)
}

1 Division of Infectious Diseases and Hospital Epidemiology, University Hospital Zürich, University of Zürich, Zürich, Switzerland, 2 Institute of Medical Virology, Swiss National Center for Retroviruses, University of Zürich, Zürich, Switzerland, 3 Laboratory of Virology, Geneva University Hospitals, Geneva, Switzerland, 4 Department Biomedicine, Haus Peterplatz, University of Basel, Basel, Switzerland, 5 Division of Immunology and Allergy, University Hospital Lausanne, Lausanne, Switzerland, 6 Division of Infectious Diseases, Clinic for Infectious Diseases, University Hospital Berne, University of Berne, Berne, Switzerland, 7 Division of Infectious Diseases, University Hospitals Geneva, Geneva, Switzerland, 8 Infectious Diseases Service, University Hospital Lausanne, Lausanne, Switzerland, 9 Division of Infectious Diseases and Hospital Epidemiology, University Hospital Basel, Basel, Switzerland, 10 Division of Infectious Diseases, Cantonal Hospital St. Gallen, St. Gallen, Switzerland, 11 Regional Hospital Lugano, Lugano, Switzerland

\section{Abstract}

Background: The accumulation of mutations after long-lasting exposure to a failing combination antiretroviral therapy (cART) is problematic and severely reduces the options for further successful treatments.

Methods: We studied patients from the Swiss HIV Cohort Study who failed CART with nucleoside reverse transcriptase inhibitors (NRTIs) and either a ritonavir-boosted $\mathrm{PI}(\mathrm{PI} / \mathrm{r}$ ) or a non-nucleoside reverse transcriptase inhibitor (NNRTI). The loss of genotypic activity $<3,3-6,>6$ months after virological failure was analyzed with Stanford algorithm. Risk factors associated with early emergence of drug resistance mutations ( $<6$ months after failure) were identified with multivariable logistic regression.

Results: Ninety-nine genotypic resistance tests from PI/r-treated and 129 from NNRTI-treated patients were analyzed. The risk of losing the activity of $\geq 1$ NRTls was lower among PI/r- compared to NNRTI-treated individuals $<3,3-6$, and $>6$ months after failure: $8.8 \%$ vs. $38.2 \%(p=0.009), 7.1 \%$ vs. $46.9 \%(p<0.001)$ and $18.9 \%$ vs. $60.9 \%(p<0.001)$. The percentages of patients who have lost $\mathrm{Pl} / \mathrm{r}$ activity were $2.9 \%, 3.6 \%$ and $5.4 \%<3,3-6,>6$ months after failure compared to $41.2 \%, 49.0 \%$ and $63.0 \%$ of those who have lost NNRTI activity (all $p<0.001$ ). The risk to accumulate an early NRTI mutation was strongly associated with NNRTI-containing CART (adjusted odds ratio: 13.3 (95\% Cl: 4.1-42.8), $\mathrm{p}<0.001$ ).

Conclusions: The loss of activity of PIs and NRTIs was low among patients treated with PI/r, even after long-lasting exposure to a failing CART. Thus, more options remain for second-line therapy. This finding is potentially of high relevance, in particular for settings with poor or lacking virological monitoring.

Citation: Scherrer AU, Böni J, Yerly S, Klimkait T, Aubert V, et al. (2012) Long-Lasting Protection of Activity of Nucleoside Reverse Transcriptase Inhibitors and Protease Inhibitors (PIs) by Boosted PI Containing Regimens. PLoS ONE 7(11): e50307. doi:10.1371/journal.pone.0050307

Editor: Joseph S. Pagano, The University of North Carolina at Chapel Hill, United States of America

Received August 1, 2012; Accepted October 18, 2012; Published November 26, 2012

Copyright: (c) 2012 Scherrer et al. This is an open-access article distributed under the terms of the Creative Commons Attribution License, which permits unrestricted use, distribution, and reproduction in any medium, provided the original author and source are credited.

Funding: This work was supported in the framework of the Swiss HIV Cohort Study, by the Swiss National Science Foundation (SNF grant \#33CS30-134277). Further support was provided by SNF grant \# 3247B0-112594 (to HFG, SY and BL), SNF grant \# 324730-130865 and the SHCS projects \# 470, 528, 569, 683, the SHCS Research Foundation, the European Community's Seventh Framework Programme (grant FP7/2007-2013), under the Collaborative HIV and Anti-HIV Drug Resistance Network (CHAIN; grant 223131), and by a further research grant of the Union Bank of Switzerland, in the name of a donor to HFG, and an unrestricted research grant from Tibotec, Switzerland, to HFG and from Gilead, Switzerland, to HFG. The funders had no role in study design, data collection and analysis, decision to publish, or preparation of the manuscript.

Competing Interests: HFG has been an adviser and/or consultant for the following companies: GlaxoSmithKline, Abbott, Novartis, Boehringer Ingelheim, Roche, Tibotec and Bristol-Myers Squibb, and has received unrestricted research and educational grants from Roche, Abbott, Bristol-Myers Squibb, GlaxoSmithKline, Gilead, Tibotec and Merck Sharp \& Dohme (all money went to institution). SY has participated in advisory boards of BMS and Tibotec, and has received travel grants from GSK and MSD. TK served as advisor for BMS and Pfizer and has received travel grants from Abbott and Pfizer. EB has been adviser and/or consultant for Gilead, Merck Sharp \& Dohme, Janssen, ViiV, and Bristol-Myers Squibb. He also received travel grants from Abbott, Gilead, Merck Sharp \& Dohme, Janssen, and ViiV. All others reported no conflict of interest.

*E-mail: alexandra.scherrer@usz.ch

\section{Introduction}

The emergence of drug resistance is one of the major threats to successful antiretroviral therapy of infection with human immunodeficiency virus-1 (HIV-1) [1]. HIV-1 cannot be eradicated with today's antiretroviral treatment. The aim of therapy is thus to reduce morbidity and mortality by long-term inhibition of HIV-1 replication. Combination antiretroviral therapy (cART) is highly effective but viruses may start replicating if drug levels are too low 
(e.g. due to patients poor adherence or drug-drug interaction), concurrent infections or recent vaccinations. In these situations drug resistance mutations can accumulate [2-7]. To avoid longlasting episodes of viral replication under cART and to detect a virological failure early, it is recommended to regularly monitor plasma viral load levels [8,9]. However, in resource-limited settings the technical equipment, health care infrastructure and financial capacity are often lacking. Monitoring of cART is therefore often solely based on the measurements of CD4 cell counts. However, monitoring of treatment success by CD4 cell counts results in a significant delay to detecting treatment failure when compared to viral load monitoring and results in a higher burden of mutations $[10,11]$. The accumulation of drug resistanceassociated mutations reduces the options for subsequent successful second-line treatment dramatically. Therefore, it is important to identify cART combinations that result in long-lasting protection of the antiretroviral activity and to minimize the emergence of drug resistance mutations even if patients need to stay extended periods on a failing therapy [12].

We aimed to study the loss of genotypic activity at different time points after virological failure and the accumulation of mutations. We further sought to identify risk factors for early emergence of mutations and we aimed to describe antiretroviral treatments with a long-lasting protection of the genotypic activity after virological failure. To answer these questions, we used data from the Swiss HIV Cohort (SHCS) and the SHCS drug resistance database and compared sequences from genotypic drug resistance tests that were performed after patients had failed first-line cART.

\section{Methods}

\section{Ethics statement}

The SHCS has been approved by the following ethical committees of all participating institutions: Kantonale Ethikkommission Bern; Ethikkommission beider Basel; comité d'éthique du département de médicine de Hôpitaux Universitaires de Genève; commission d'éthique de la recherche clinique, Lausanne; comitato etico cantonale, Bellinzona; Ethikkommission des Kanton St.Gallens; and Ethik-Kommission Zürich, all Switzerland. Written informed consent has been obtained from all participants [13].

\section{Study population}

We compared genotypic drug resistance tests from individuals included in the SHCS who failed first-line cART. The SHCS is a nationwide, multicenter, clinic-based cohort with continuous enrolment and semi-annual study visits. The last considered follow-up was the 18 October 2011. The SHCS drug resistance database is linked to the SHCS and includes $>14,000$ sequences from genotypic drug resistance tests performed by one of the four authorized laboratories in Switzerland [14]. Sequences are stored in SmartGene's (Zug, Switzerland) Integrated Database Network System (IDNS version 3.6.6).

\section{Patient selection and statistical analysis}

We did a cross-sectional analysis and restricted our study to individuals who started first-line cART with nucleoside reverse transcriptase inhibitors (NRTIs) and either a ritonavir-boosted protease inhibitor $(\mathrm{PI} / \mathrm{r})$ or a non-nucleoside reverse transcriptase inhibitor (NNRTI) and who had a genotypic drug resistance test performed after virological failure but before treatment change to second-line cART. A treatment failure was defined if at least one HIV-1 RNA was detectable ( $\geq 50$ copies $/ \mathrm{mL}$ ) after previous suppression $(<50$ copies $/ \mathrm{mL})$ or when individuals did not respond to the first-line cART for at least 180 days (no viral load $<50$ copies $/ \mathrm{mL}$ ). These individuals are further termed non-responders. If cART was changed before 90 days or if the last viral load during cART was undetectable, it was assumed that the treatment change was due to toxicity reasons and the next treatment was considered for analysis.

We calculated the time with replicating virus after virological failure until the resistance test was performed. The time with replicating virus was defined as the time period when patients had detectable viral loads ( $>50$ copies $/ \mathrm{mL}$ ). If viral loads changed between two measurements from undetectable to detectable or vice versa, it was assumed that viruses were replicating half of the time. We grouped individuals with $<3,3-6$ and $>6$ months with replicating virus.

Characteristics were compared with Fishers exact test (categorical variables) and Wilcoxon rank-sum test (continuous variables).

The loss of genotypic activity was estimated using the Stanford algorithm (version 6.1.1). The activity of a drug was defined as lost when the Stanford penalty score was $\geq 30$ (http://hivdb.stanford. edu/). Drug resistance associated mutations were defined by IASUSA [15]. Minor PI mutations were not considered for analysis. Thymidine-analogue mutations (TAMs) were categorized in TAM 1 (M41L, L210W, T215Y) and TAM 2 (D67N, K70R, T215F, $\mathrm{K} 219 \mathrm{E} / \mathrm{Q}$

We identified risk factors for an early accumulation of mutations ( $<6$ months with replicating viruses). We performed univariable and multivariable logistic regression analyses. The following variables were included in the multivariable model: sex, transmission group, age, subtype, square root CD4 cell and viral load at the time when the resistance test was performed, NRTI treatment, $\mathrm{PI} / \mathrm{r}$ or NNRTI use, and the year of cART initiation. Likelihood ratio tests did not indicate significant departures from linearity for continuous variables.

Adherence is an additional potential confounder. Self-reported adherence is documented only since May 2003 in the SHCS, therefore a sensitivity analysis including adherence data was performed with patients who failed cART after this date [16].

Not only the time with replicating virus but also the viral load might be predictive for the number of emerging mutations, therefore copy-years viremia was used in a sensitivity analysis instead of the time with replicating virus [17]. Copy-years viremia is a way to express the amount of exposure an individual has had to the virus over a period of time (akin to pack-years of smoking). The mean viral load of two successive measurements is multiplied by the time they are apart.

Statistical analyses were performed with Stata 11 SE software (StataCorp, College Station, TX, USA). All confidence intervals were two-sided, and the level of significance was set at 0.05.

\section{Results}

\section{Baseline characteristics}

We included 129 patients with a virological failure on a NNRTI-containing and 99 on a PI/r-containing cART (Table 1). The fraction of non-responders (patients who did not reach $<50$ copies $/ \mathrm{mL}$ ) was $20.2 \%$ and $22.2 \%(\mathrm{p}=0.745)$, respectively. Most baseline characteristics were similar between groups, although PI/ r-treated patients started cART later (median: 2007 vs. 2004, $\mathrm{p}<0.001$ ). The co-administered NRTIs varied slightly. The most commonly used NRTI combination in individuals treated with NNRTIs was zidovudine (AZT) and lamivudine (3TC) (41.1\%). Most PI/r-treated patients received tenofovir (TDF) with either 3TC or emtricitabine (FTC) $(45.5 \%, \mathrm{p}=0.061)$. The median time with replicating virus was 144.5 days (IQR: 87.5-233) and 141 


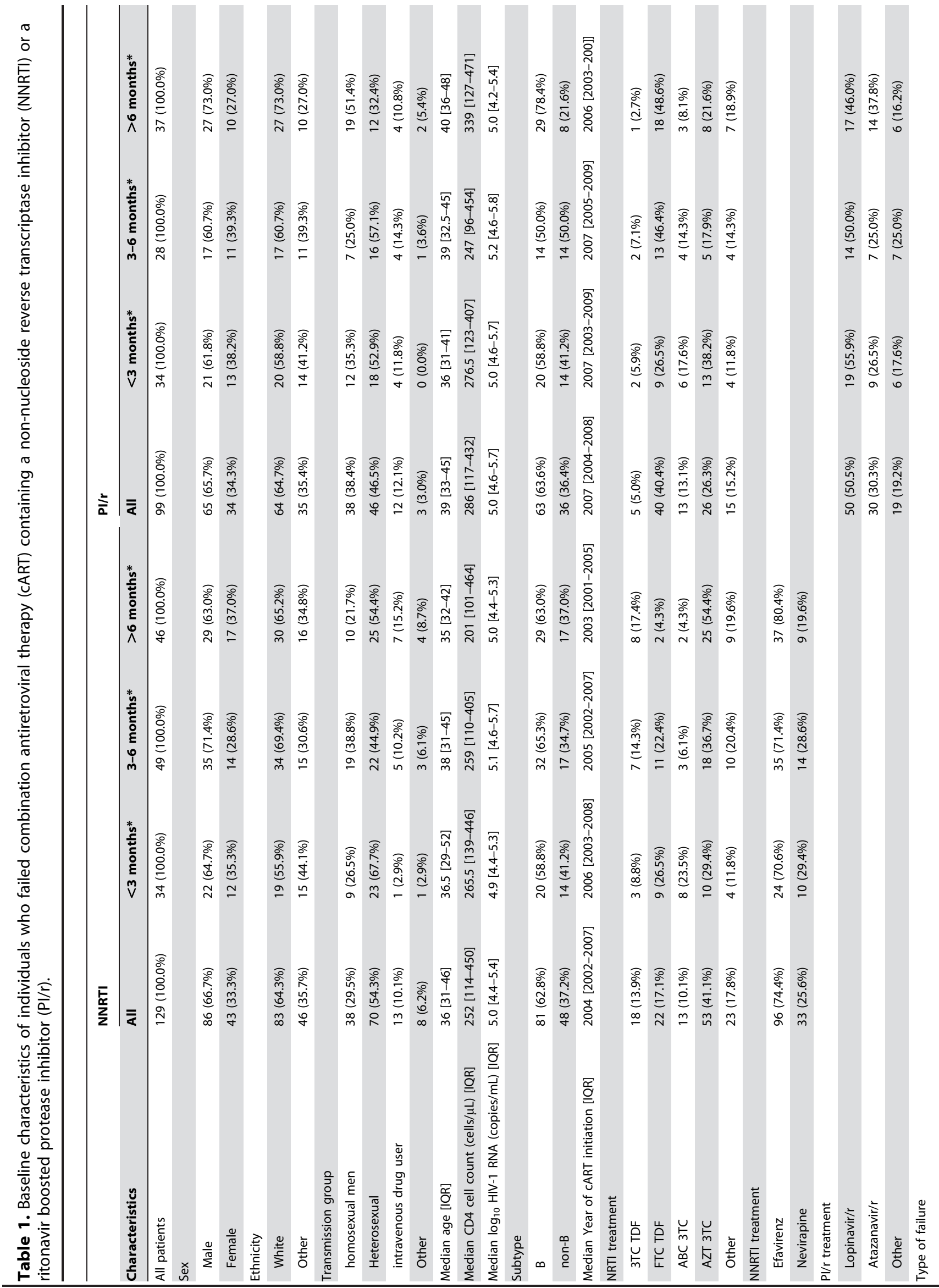




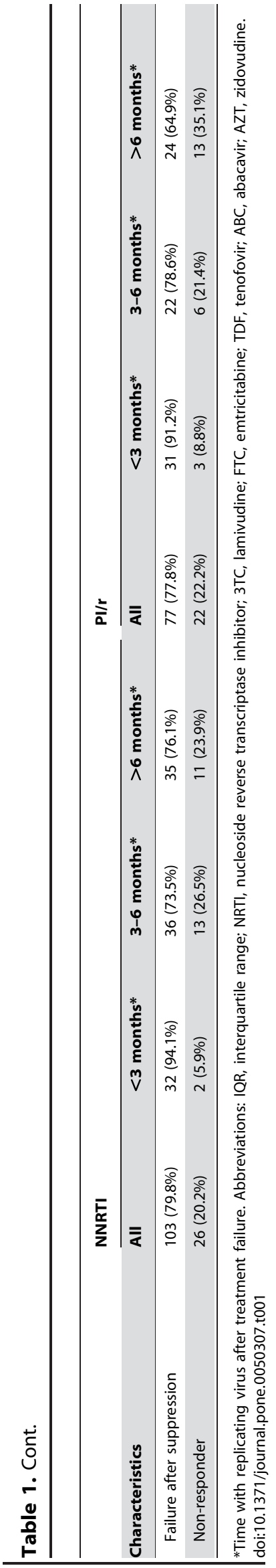

days (IQR: 65-268) for NNRTI- and PI/r-treated patients $(\mathrm{p}=0.573)$, respectively. Individuals were categorized by the time the resistance test was performed after virological failure: 34 and 34, 49 and 28, 46 and 37 treated with NNRTI or PI, respectively, had a resistance test performed after $<3,3-6$ and $>6$ months with replicating virus. The median time with replicating virus in the category $>6$ months was similar between groups: 277.3 days (IQR: 226-506.5) and 292 days (IQR: 234.5-428) for NNRTIand $\mathrm{PI} / \mathrm{r}$-treated patients $(\mathrm{p}=0.916)$, respectively.

The median viral load at the time when the genotypic resistance test was performed was considerably higher among patients treated with a NNRTI compared to a PI/r (HIV-1 RNA: $\log _{10} 3.5$ copies/mL [IQR: 2.8-4.6] vs. $\log _{10} 2.8$ copies/mL [IQR: $2.3-$ 3.6], $\mathrm{p}<0.001)$. In addition, the copy-years viremia was also substantially higher in patients treated with NNRTI (641.2 years * copies/mL [IQR: 114.5-8348.1], p<0.001) compared to PI/r (216.9 years * copies/mL [IQR: 33.9-1454.6]).

The numbers of transmitted NRTI mutations were similar in NNRTI- and PI/r treated individuals. A resistance test had been performed prior to any cART initiation among 146 of 228 individuals. The prevalence of transmitted NRTI mutations was $1.5 \%$ and $5.0 \%$ in $\mathrm{PI} / \mathrm{r}$ and NNRTI-treated individuals $(p=0.378)$, respectively. Restricting the analysis to patients with known baseline resistance data and without transmitted drug resistance mutations did not alter conclusions (data not shown).

Adherence data was available for 157 of 169 (92.9\%) individuals who failed cART after May 2003. Adherence was similar between patients treated with PI/r- or NNRTI-containing cART: $76.9 \%$ and $79.8 \%(p=0.503)$ never missed a drug dose six months before reporting adherence.

\section{Loss of genotypic activity of NRTIs}

The loss of genotypic activity of NRTIs was considerably higher in patients treated with NNRTIs compared to PI/r. The loss of genotypic activity of NRTIs was already very high $<3$ months after failure when patients had been treated with NNRTIs (38.2\%) whereas PI/r-treated patients rarely accumulated NRTI mutations in this time period $(8.8 \%, \mathrm{p}=0.009)$. The loss of genotypic activity of NRTIs remained considerably higher among patients treated with NNRTIs compared to PI/r also after long-lasting exposure to the failing regimen: $46.9 \%$ vs. $7.1 \% \quad(\mathrm{p}<0.001)$ between 3-6 months and $60.9 \%$ vs. $18.9 \%(\mathrm{p}<0.001)$ after $>6$ months with replicating virus. The loss of activity of more than one NRTI was quite rare in the PI/r group for all time points: $2.9 \%$, $7.1 \%, 0 \%$ when compared to the NNRTI group: $14.7 \%$ $(p=0.197), 14.3 \%(p=0.474), 21.7 \%(p=0.002)$ at $<3,3-6$ and $>6$ months after failure (Figure $1 \mathrm{~A}$ ).

We excluded non-responders $(n=48)$ from a sensitivity analysis. The results remained more favorable for the PI/r group: $37.5 \% \mathrm{vs}$. $9.7 \%(p=0.016), 38.9 \%$ vs. $9.1 \%(p=0.016), 57.1 \%$ vs. $16.7 \%$ $(\mathrm{p}=0.003)$ NNRTI- and PI-treated individuals lost the activity of $\geq 1$ NRTI after $<3,3-6,>6$ months with replicating virus. Results were confirmed if the analysis was restricted to the 113 patients who truly failed first-line cART (patients who changed a successful cART regimen due to toxicity reasons were excluded) although the sample size was reduced: $23.1 \%$ vs. $38.5 \%$ $(p=0.673), \quad 8.3 \% \quad$ vs. $48.0 \% \quad(p=0.027), 22.2 \%$ vs. $65.6 \%$ $(\mathrm{p}=0.007)$ after $<3,3-6,>6$ months with replicating virus lost the activity of $\geq 1$ NRTI in the $\mathrm{PI} / \mathrm{r}$ and NNRTI group, respectively. The treatment with more potent NRTI combinations could be a potential explanation for the lower accumulation of NRTI mutations. PI/r-treated individuals received more often the modern and well tolerated NRTI combination TDF/FTC (40.4\%) compared to NNRTI-treated individuals (17.1\%). In a sensitivity 

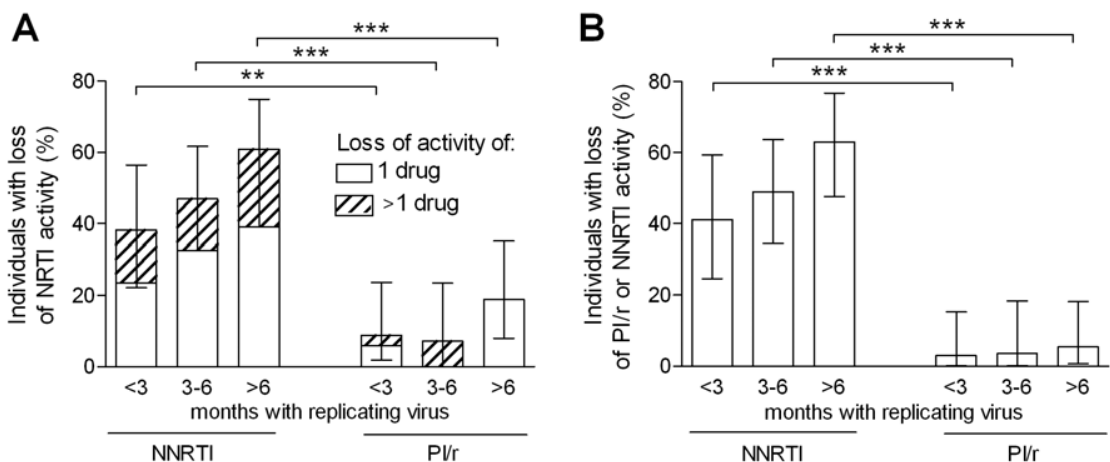

Figure 1. Loss of genotypic activity. Loss of genotypic activity among patients treated with non-nucleoside reverse transcriptase inhibitors (NNRTIs) or ritonavir-boosted protease inhibitors (PI/r). A) Loss of activity of 1 NRTI (open bar section) or $>1$ NRTIs (hatched bar section). B) Loss of activity of a NNRTI or PI/r. 95\% confidence intervals are indicated. ${ }^{* * *}$ Fisher exact $p<0.001,{ }^{* *} p<0.01$.

doi:10.1371/journal.pone.0050307.g001

analysis, we only included patients who were treated with the TDF/FTG NRTI combination $(n=62)$. Also in this sub-group, the loss of activity of $\geq 1$ NRTI was higher in NNRTI compared to $\mathrm{PI} / \mathrm{r}$-treated individuals, $26.1 \%$ compared to $5 \%(\mathrm{p}=0.043)$, respectively.

Higher copy-years viremia among patients treated with NNRTI would be a potential explanation for faster accumulation of mutations. We repeated the analysis and classified patients in 3 groups based on the copy-years viremia instead of the time with replicating virus. Each group contained 76 patients with copyyears viremia $<115,115-1491,>1491$ years $*$ copies $/ \mathrm{mL}$. The loss of genotypic activity of $\geq 1$ NRTIs was $60.6 \%$ vs. $7.0 \%$ $(p<0.001), 51.2 \%$ vs. $15.2 \%(p=0.002)$ and $41.5 \%$ vs. $17.4 \%$ $(\mathrm{p}=0.064)$ in group 1 to 3 among NNRTI and PI/r treated individuals, respectively. These findings demonstrate that the higher loss of activity in NNRTI-treated patients cannot solely be explained by a more intense viral replication.

\section{Loss of genotypic activity of PIs and NNRTIs}

The loss of genotypic activity of NNRTIs occurred very early after treatment failure whereas the emergence of $\mathrm{PI} / \mathrm{r}$ resistance was very rare even after long-lasting exposure to failing cART. The percentage of patients who have lost PI/r activity was $2.9 \%$, $3.6 \%, 5.4 \%<3,3-6$ and $>6$ months compared to $41.2 \%, 49.0 \%$, $63.0 \%$ of those who have lost NNRTI activity (all p<0.001) (Figure 1 B). Results were similar when individuals were categorized according to copy-years viremia strata: $7.0 \%$ vs. $57.6 \%, 0 \%$ vs. $44.2 \%, 4.4 \%$ vs. $54.7 \%$ (all $\mathrm{p}<0.001$ ).

Excluding non-responders or studying exclusively patients who failed first-line treatment confirmed these results (data not shown).

\section{Emerging mutations}

The most common cause for the loss of genotypic activity of NRTIs was the emergence of M184V. It occurred in $36.4 \%$ of NNRTI- and in only $9.1 \%$ of PI/r-treated individuals $(\mathrm{p}<0.001)$. The prevalence of additional NRTI mutations was also much higher in the NNRTI when compared to the PI/r group: K65R $10.9 \%$ vs. $1.0 \%$ ( $p=0.003)$, M184I $7.0 \%$ vs. $1.0 \% \quad(p=0.029)$, $\mathrm{T} 215 \mathrm{Y} 5.4 \%$ vs. $1.0 \%(\mathrm{p}=0.072)$. All other mutations had a prevalence of $<5 \%$ in both groups (Figure 2).

The loss of NNRTI activity was mainly caused by the emergence of $\mathrm{K} 103 \mathrm{~N}$ (27.9\%), Y181C (10.9\%) or G190A $(6.2 \%)$. The most common PI mutations in PI/r-treated individuals were L90M (3.0\%), I84V (2.0\%) and M46I (2.0\%).
Risk factors for the early emergence of NRTI mutations

Exposure to NNRTIs was the major risk factor for an early accumulation of NRTI mutations. The odds ratio (OR) was 10.6 (95\% CI: 3.9-29.1) and 13.3 (95\% CI: 4.1-42.8) in univariable and multivariable models, respectively. High CD4 cell count at the time of the resistance test was the only other factor that had a weak association with a later accumulation of NRTI mutations (univariable OR: 0.9 (95\% CI: 0.9-1.0), multivariable OR: 0.9 (95\% CI: $0.8-1.0)$. No other factor was significantly associated in the multivariable model (Table 2).

\section{Discussion}

We showed that cART containing PI/r results in a long-lasting protection of the activity of NRTIs and PI/r during sustained viral

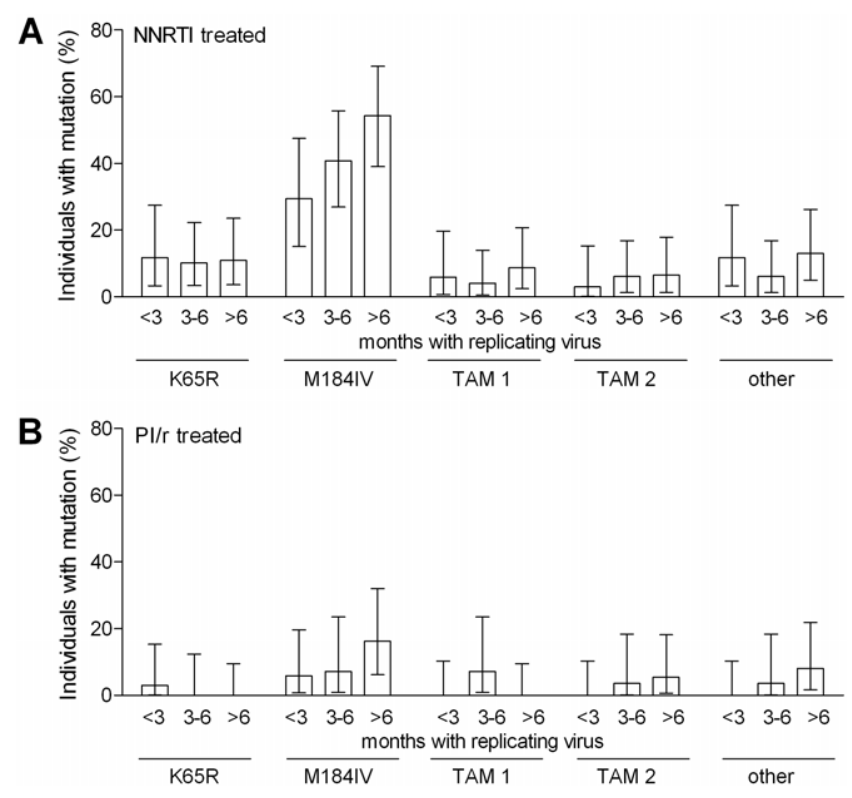

Figure 2. Emergence of mutations. Accumulation of nucleoside reverse transcriptase inhibitor (NRTI) mutations after virological failure on combination antiretroviral therapy containing A) non-nucleoside reverse transcriptase inhibitors (NNRTIs) or B) ritonavir-boosted protease inhibitors $(\mathrm{PI} / \mathrm{r}): \mathrm{K} 65 \mathrm{R}, \mathrm{M} 184 \mathrm{I} / \mathrm{V}$, thymidine analogue mutations (TAM) 1 or 2, and other NRTI mutations defined by IAS-USA [15]. 95\% confidence intervals are indicated.

doi:10.1371/journal.pone.0050307.g002 
Table 2. Univariable and multivariable logistic regression analyzing risk factors for an early ( $<6$ months with replicating viruses) emergence of $\geq 1$ nucleoside reverse transcriptase (NRTI) mutations ( $n=145$ ).

\begin{tabular}{|c|c|c|c|c|c|}
\hline Characteristics & $\begin{array}{l}\text { Individuals with } \geq 1 \text { NRTI } \\
\text { mutation/failures (\%) }\end{array}$ & $\begin{array}{l}\text { Univariable odds } \\
\text { ratio }(95 \% \mathrm{Cl})\end{array}$ & $\mathbf{P}$ & $\begin{array}{l}\text { Multivariable odds } \\
\text { ratio }(95 \% \mathrm{Cl})\end{array}$ & $\mathbf{P}$ \\
\hline \multicolumn{6}{|l|}{ Sex } \\
\hline male & 30/95 (31.6\%) & 1 (Ref) & & 1 (Ref) & \\
\hline female & $15 / 50(30.0 \%)$ & $0.9(0.4-2.0)$ & 0.845 & $1.3(0.5-3.5)$ & 0.663 \\
\hline \multicolumn{6}{|l|}{ Transmission group } \\
\hline non-IDU & 39/131 (29.8\%) & 1 (Ref) & & 1 (Ref) & \\
\hline IDU & 6/14 (42.9\%) & $1.8(0.6-5.4)$ & 0.319 & $2.4(0.5-12.0)$ & 0.274 \\
\hline Age & & $1.0(0.9-1.0)$ & 0.343 & $1.0(0.9-1.0)$ & 0.175 \\
\hline \multicolumn{6}{|l|}{ Subtype } \\
\hline non-B & 15/59 (25.4\%) & 1 (Ref) & & 1 (Ref) & \\
\hline B & $30 / 86(34.9 \%)$ & $1.6(0.8-3.3)$ & 0.228 & $1.6(0.6-4.4)$ & 0.401 \\
\hline$\sqrt{\mathrm{CD} 4}$ cells $/ \mu \mathrm{L}$ & & $0.9(0.9-1.0)$ & 0.014 & $0.9(0.8-1.0)$ & 0.024 \\
\hline $\log _{10}$ HIV RNA & & $1.5(1.1-2.1)$ & 0.013 & $1.0(0.6-1.5)$ & 0.907 \\
\hline \multicolumn{6}{|l|}{ NRTI backbone } \\
\hline 3TC TDF & $8 / 14(57.1 \%)$ & 1 (Ref) & & 1 (Ref) & \\
\hline FTC TDF & 8/42(19.1\%) & $0.2(0.0-0.7)$ & 0.009 & $0.3(0.1-1.8)$ & 0.195 \\
\hline $\mathrm{ABC} 3 \mathrm{TC}$ & 4/21(19.1\%) & $0.2(0.0-0.8)$ & 0.025 & $0.2(0.0-1.3)$ & 0.093 \\
\hline AZT 3TC & 15/46(32.6\%) & $0.4(0.1-1.2)$ & 0.105 & $0.4(0.1-1.9)$ & 0.222 \\
\hline other & 10/22(45.5\%) & $0.6(0.2-2.4)$ & 0.495 & $0.5(0.1-2.9)$ & 0.460 \\
\hline \multicolumn{6}{|l|}{ Second drug class } \\
\hline $\mathrm{Pl} / \mathrm{r}$ & $5 / 62(8.1 \%)$ & 1 (Ref) & & 1 (Ref) & \\
\hline NNRTI & 40/83 (48.2\%) & $10.6(3.9-29.1)$ & $<0.001$ & $14.3(4.3-47.5)$ & $<0.001$ \\
\hline Year of cART start & & $0.9(0.8-1.0)$ & 0.010 & $1.0(0.8-1.2)$ & 0.698 \\
\hline
\end{tabular}

Abbreviation: $\mathrm{Cl}$, confidence interval; IDU, intravenous drug user; FTC, emtricitabine; 3TC, lamivudine; ABC, abacavir; AZT, zidovudine; TDF, tenofovir; PI/r, ritonavirboosted protease inhibitor; NNRTI, non-nucleoside reverse transcriptase inhibitor.

doi:10.1371/journal.pone.0050307.t002

replication under therapy. In contrast, if patients are treated with NNRTI-based cART, NRTI mutations emerge much earlier and in larger numbers. These findings are of importance both, for resource-rich and resource-limited settings. In resource-rich settings, treatment failures are usually diagnosed quite early because of frequent viral load monitoring. In resource-limited settings patients often stay a long time on a failing regimen due to lacking or only infrequent viral load monitoring. In both situations, more options remain for second-line treatment if patients receive a $\mathrm{PI} / \mathrm{r}$-based cART as first-line therapy.

Previous randomized and observational studies showed that the failure rate between PI/r and NNRTI is comparable in most cases but fewer mutations emerge when patients fail a $\mathrm{PI} / \mathrm{r}$ treatment [14,18-21]. Mainly the activity of $\mathrm{PI} / \mathrm{r}$ is well protected but also the activity of NRTIs [21-25]. In extension to these earlier data, we demonstrated in our study that this effect is long-lasting. After more than 6 months sustained viral replication on PI/r-containing cART, the loss of activity of $\geq 1$ NRTI is only $18.9 \%$ compared to $60.9 \%$ on NNRTI-containing cART. This finding is of particular interest for resource-limited settings without virological monitoring where high numbers of NRTI mutations, mainly M184V, and NNRTI mutations are common in first-line failures treated with NNRTI-containing cART [26-28]. The number of accumulating mutations can be reduced when virological monitoring is performed [10,29]. However, in many settings infrastructure and costs do not allow virological monitoring at regular intervals [12], therefore the use of $\mathrm{PI} / \mathrm{r}$ as first-line therapy might be an interesting alternative in order to save more options for secondline treatment. Although drug resistance is an important factor to be considered, co-formulations, simplicity of administration, costs, drug-drug interactions, toxicity and adverse events need also to be taken into account for the choice of first-line treatment [8].

In general, it is astonishing how few mutations were observed overall in the 228 patients of the study who have failed therapy. Only $43 \%$ of patients had any drug resistance-associated mutation detected [21]. Missing drug pressure due to poor adherence could be a possible explanation for the low prevalence of mutations but it is probably not the major reason because $>75 \%$ of patients reported to have an excellent adherence. Nevertheless, the prevalence of resistance might be underestimated. Currently used genotypic resistance tests have a population detection limit of only $\sim 20 \%$. Additional resistant virus variants might be present at lower levels [30-33]. The late and rare occurrence of $\mathrm{PI} / \mathrm{r}$ mutations can be explained by their high genetic barrier compared to NNRTIs [34]. However, the mechanism explaining the lack of resistance to co-administered NRTIs remains unknown. It can be speculated that the two drug classes may have different activities in different anatomical compartments [35], with regards to free versus cell-cell virus transmission [36] so that the activity of PI/r might be sufficient to suppress NRTI resistant strains to undetectable levels [21]. It could also be possible that NNRTIs, as they target the same gene as NRTIs, might select for yet unidentified compensatory mutations in the RT connection-, respectively, RNase H-domain of the pol gene [37,38], subse- 
quently leading to more rapid emergence of NRTI mutations $[39,40]$. In theory, the presence of minority variants harboring NNRTI- or NRTI-drug resistant mutations, which have been detected in drug naive HIV-1 infected patients, could have a more severe impact in a regimen that contains a "low genetic barrier" drug rather than a PI/r. This aspect cannot be excluded in the present study. $[32,41]$. Poorer adherence in the PI/r-treated group could also possibly explain the differences (no selective drug pressure from NRTIs) but adherence was excluded as potential bias in a sensitivity analysis. In addition, different NRTI backbones in PI/r- and NNRTI-treated individuals might have influenced our results $[42,43]$. To disprove this concern, we performed a sensitivity analysis only including patients with a TDF/FTC backbone and we adjusted the logistic regression for the NRTI backbone.

Although our study initially considered 5959 patients who started first-line cART, only 228 individuals qualified for our study. The sample size was too small to compare different treatment regimens in more detail. Unfortunately, sufficient longitudinal resistance data from our patients were not available; otherwise dynamics of evolution of individual drug resistance

\section{References}

1. Hogg RS, Bangsberg DR, Lima VD, Alexander C, Bonner S, et al. (2006) Emergence of drug resistance is associated with an increased risk of death among patients first starting HAART. PLoS Med 3: e356.

2. Cozzi-Lepri A, Phillips AN, Ruiz L, Clotet B, Loveday C, et al. (2007) Evolution of drug resistance in HIV-infected patients remaining on a virologically failing combination antiretroviral therapy regimen. AIDS 21: 721-732.

3. Hoffmann CJ, Charalambous S, Sim J, Ledwaba J, Schwikkard G, et al. (2009) Viremia, resuppression, and time to resistance in human immunodeficiency virus (HIV) subtype C during first-line antiretroviral therapy in South Africa. Clin Infect Dis 49: 1928-1935.

4. Napravnik S, Edwards D, Stewart P, Stalzer B, Matteson E, et al. (2005) HIV-1 drug resistance evolution among patients on potent combination antiretroviral therapy with detectable viremia. J Acquir Immune Defic Syndr 40: 34 40.

5. Reynolds SJ, Kityo C, Mbamanya F, Dewar R, Ssali F, et al. (2009) Evolution of drug resistance after virological failure of a first-line highly active antiretroviral therapy regimen in Uganda. Antivir Ther 14: 293-297.

6. Kantor R, Shafer RW, Follansbee S, Taylor J, Shilane D, et al. (2004) Evolution of resistance to drugs in HIV-1-infected patients failing antiretroviral therapy. AIDS 18: 1503-1511.

7. Wittkop L, Gunthard HF, de Wolf F, Dunn D, Cozzi-Lepri A, et al. (2011) Effect of transmitted drug resistance on virological and immunological response to initial combination antiretroviral therapy for HIV (EuroCoord-CHAIN joint project): a European multicohort study. Lancet Infect Dis 11: 363-371.

8. Thompson MA, Aberg JA, Cahn P, Montaner JS, Rizzardini G, et al. (2010) Antiretroviral treatment of adult HIV infection: 2010 recommendations of the International AIDS Society-USA panel. JAMA 304: 321-333.

9. Petersen ML, van der Laan MJ, Napravnik S, Eron JJ, Moore RD, et al. (2008) Long-term consequences of the delay between virologic failure of highly active antiretroviral therapy and regimen modification. AIDS 22: 2097-2106.

10. Gupta RK, Hill A, Sawyer AW, Cozzi-Lepri A, von Wyl V, et al. (2009) Virological monitoring and resistance to first-line highly active antiretroviral therapy in adults infected with HIV-1 treated under WHO guidelines: a systematic review and meta-analysis. Lancet Infect Dis 9: 409-417.

11. Rawizza HE, Chaplin B, Meloni ST, Eisen G, Rao T, et al. (2011) Immunologic criteria are poor predictors of virologic outcome: implications for HIV treatment monitoring in resource-limited settings. Clin Infect Dis 53: 1283-1290.

12. Phillips AN, Pillay D, Miners AH, Bennett DE, Gilks CF, et al. (2008) Outcomes from monitoring of patients on antiretroviral therapy in resource-limited settings with viral load, CD4 cell count, or clinical observation alone: a computer simulation model. Lancet 371: 1443-1451.

13. Schoeni-Affolter F, Ledergerber B, Rickenbach M, Rudin C, Gunthard HF, et al. (2010) Cohort profile: the Swiss HIV Cohort study. Int J Epidemiol 39: 1179 1189 .

14. von Wyl V, Yerly S, Boni J, Burgisser P, Klimkait T, et al. (2007) Emergence of HIV-1 drug resistance in previously untreated patients initiating combination antiretroviral treatment: a comparison of different regimen types. Arch Intern Med 167: 1782-1790.

15. Johnson VA, Calvez V, Gunthard HF, Paredes R, Pillay D, et al. (2011) 2011 update of the drug resistance mutations in HIV-1. Top Antivir Med 19: 156164.

16. Glass TR, De Geest S, Weber R, Vernazza PL, Rickenbach M, et al. (2006) Correlates of self-reported nonadherence to antiretroviral therapy in HIV- mutations could have been investigated in more detail. In addition, we cannot exclude that there are resistance associated mutations outside the sequenced region. No phenotypic resistance tests were available that could prove that viruses which do not harbor any mutations are really sensitive to the drugs.

In conclusion, PI/r containing cART leads to long-lasting protection of the activity of NRTIs and PI/r despite ongoing viral replication after virological failure. Accumulation of drug resistance mutations against all three drugs of the regimen is slower and less frequent when compared to NNRTI-containing regimens, thus retaining more options for second-line therapy. These findings are of high relevance for settings, which lack the opportunities for regular virological monitoring and where the use of PI/r as first-line therapies should be considered.

\section{Author Contributions}

Conceived and designed the experiments: AUS BL HFG. Performed the experiments: JB SY TK VA. Analyzed the data: AUS. Contributed reagents/materials/analysis tools: HF AC MC LE PLV EB. Wrote the paper: AUS HFG.

infected patients: the Swiss HIV Cohort Study. J Acquir Immune Defic Syndr 41: 385-392.

17. Cole SR, Napravnik S, Mugavero MJ, Lau B, Eron JJ Jr, et al. (2010) Copvyears viremia as a measure of cumulative human immunodeficiency virus viral burden. Am J Epidemiol 171: 198-205.

18. Shafer RW, Smeaton LM, Robbins GK, De Gruttola V, Snyder SW, et al. (2003) Comparison of four-drug regimens and pairs of sequential three-drug regimens as initial therapy for HIV-1 infection. N Engl J Med 349: 2304-2315.

19. MacArthur RD, Novak RM, Peng G, Chen L, Xiang Y, et al. (2006) A comparison of three highly active antiretroviral treatment strategies consisting of non-nucleoside reverse transcriptase inhibitors, protease inhibitors, or both in the presence of nucleoside reverse transcriptase inhibitors as initial therapy (CPCRA 058 FIRST Study): a long-term randomised trial. Lancet 368: 21252135.

20. Daar ES, Tierney C, Fischl MA, Sax PE, Mollan K, et al. (2011) Atazanavir plus ritonavir or efavirenz as part of a 3-drug regimen for initial treatment of HIV-1. Ann Intern Med 154: 445-456.

21. Gupta R, Hill A, Sawyer AW, Pillay D (2008) Emergence of drug resistance in HIV type 1-infected patients after receipt of first-line highly active antiretroviral therapy: a systematic review of clinical trials. Clin Infect Dis 47: 712-722.

22. Babiker A, Castro nee Green H, Compagnucci A, Fiscus S, Giaquinto C, et al. (2011) First-line antiretroviral therapy with a protease inhibitor versus nonnucleoside reverse transcriptase inhibitor and switch at higher versus low viral load in HIV-infected children: an open-label, randomised phase 2/3 trial. Lancet Infect Dis 11: 273-283.

23. Bartlett JA, Buda JJ, von Scheele B, Mauskopf JA, Davis EA, et al. (2006) Minimizing resistance consequences after virologic failure on initial combination therapy: a systematic overview. J Acquir Immune Defic Syndr 41: 323-331.

24. Mtambo A, Chan K, Shen A, Lima V, Hogg R, et al. (2012) Treatment limitations imposed by antiretroviral drug resistance mutations: implication for choices of first line regimens in resource-limited settings. HIV Med 13: 141-147.

25. Riddler SA, Haubrich R, DiRienzo AG, Peeples L, Powderly WG, et al. (2008) Class-sparing regimens for initial treatment of HIV-1 infection. N Engl J Med 358: 2095-2106.

26. Hosseinipour MC, van Oosterhout JJ, Weigel R, Phiri S, Kamwendo D, et al (2009) The public health approach to identify antiretroviral therapy failure: high-level nucleoside reverse transcriptase inhibitor resistance among Malawians failing first-line antiretroviral therapy. AIDS 23: 1127-1134.

27. Sungkanuparph S, Manosuthi W, Kiertiburanakul S, Piyavong B, Chumpathat $\mathrm{N}$, et al. (2007) Options for a second-line antiretroviral regimen for HIV type 1infected patients whose initial regimen of a fixed-dose combination of stavudine, lamivudine, and nevirapine fails. Clin Infect Dis 44: 447-452.

28. Murillo W, de Rivera IL, Parham L, Jovel E, Palou E, et al. (2010) Prevalence of drug resistance and importance of viral load measurements in Honduran HIVinfected patients failing antiretroviral treatment. HIV Med 11: 95-103.

29. Wallis CL, Papathanasopolous MA, Fox M, Conradie F, Ive P, et al. (2012) Low rates of nucleoside reverse transcriptase inhibitor resistance in a well-monitored cohort in South Africa on antiretroviral therapy. Antivir Ther 17: 313-320.

30. Metzner KJ, Bonhoeffer S, Fischer M, Karanicolas R, Allers K, et al. (2003) Emergence of minor populations of human immunodeficiency virus type 1 carrying the M184V and L90M mutations in subjects undergoing structured treatment interruptions. J Infect Dis 188: 1433-1443. 
31. Metzner KJ, Allers K, Rauch P, Harrer T (2007) Rapid selection of drugresistant HIV-1 during the first months of suppressive ART in treatment-naive patients. AIDS 21: 703-711.

32. Li JZ, Paredes R, Ribaudo HJ, Svarovskaia ES, Metzner KJ, et al. (2011) Lowfrequency HIV-1 drug resistance mutations and risk of NNRTI-based antiretroviral treatment failure: a systematic review and pooled analysis. JAMA 305: 1327-1335.

33. Simen BB, Simons JF, Hullsiek KH, Novak RM, Macarthur RD, et al. (2009) Low-abundance drug-resistant viral variants in chronically HIV-infected, antiretroviral treatment-naive patients significantly impact treatment outcomes. J Infect Dis 199: 693-701.

34. van de Vijver DA, Wensing AM, Angarano G, Asjo B, Balotta C, et al. (2006) The calculated genetic barrier for antiretroviral drug resistance substitutions is largely similar for different HIV-1 subtypes. J Acquir Immune Defic Syndr 41: $352-360$.

35. Letendre S, Marquie-Beck J, Capparelli E, Best B, Clifford D, et al. (2008) Validation of the CNS Penetration-Effectiveness rank for quantifying antiretroviral penetration into the central nervous system. Arch Neurol 65: 65-70.

36. Sigal A, Kim JT, Balazs AB, Dekel E, Mayo A, et al. (2011) Cell-to-cell spread of HIV permits ongoing replication despite antiretroviral therapy. Nature 477: 9598.

37. Nikolenko GN, Palmer S, Maldarelli F, Mellors JW, Coffin JM, et al. (2005) Mechanism for nucleoside analog-mediated abrogation of HIV-1 replication: balance between RNase $\mathrm{H}$ activity and nucleotide excision. Proc Natl Acad Sci U S A 102: 2093-2098.
38. Nikolenko GN, Delviks-Frankenberry KA, Palmer S, Maldarelli F, Fivash MJJr, et al. (2007) Mutations in the connection domain of HIV-1 reverse transcriptase increase 3'-azido-3'-deoxythymidine resistance. Proc Natl Acad Sci U S A 104: 317-322.

39. Yap SH, Sheen CW, Fahey J, Zanin M, Tyssen D, et al. (2007) N348I in the connection domain of HIV-1 reverse transcriptase confers zidovudine and nevirapine resistance. PLoS Med 4: e335.

40. Hachiya A, Kodama EN, Sarafianos SG, Schuckmann MM, Sakagami Y, et al. (2008) Amino acid mutation N348I in the connection subdomain of human immunodeficiency virus type 1 reverse transcriptase confers multiclass resistance to nucleoside and nonnucleoside reverse transcriptase inhibitors. J Virol 82: 3261-3270.

41. Metzner KJ, Rauch P, von Wyl V, Leemann C, Grube C, et al. (2010) Efficient suppression of minority drug-resistant HIV type 1 (HIV-1) variants present at primary HIV-1 infection by ritonavir-boosted protease inhibitor-containing antiretroviral therapy. J Infect Dis 201: 1063-1071.

42. Margot NA, Enejosa J, Cheng AK, Miller MD, McColl DJ (2009) Development of HIV-1 drug resistance through 144 weeks in antiretroviral-naive subjects on emtricitabine, tenofovir disoproxil fumarate, and efavirenz compared with lamivudine/zidovudine and efavirenz in study GS-01-934. J Acquir Immune Defic Syndr 52: 209-221.

43. Svicher V, Alteri C, Artese A, Forbici F, Santoro MM, et al. (2010) Different evolution of genotypic resistance profiles to emtricitabine versus lamivudine in tenofovir-containing regimens. J Acquir Immune Defic Syndr 55: 336-344. 